\title{
Thermodynamics of nonlinear electrodynamics black holes and the validity of weak cosmic censorship at charged particle absorption
}

\author{
Peng Wang ${ }^{1, \mathrm{a}}$, Houwen $\mathrm{Wu}^{1, \mathrm{~b}}$, Haitang Yang ${ }^{1, \mathrm{c}}$ \\ ${ }^{1}$ Center for Theoretical Physics, College of Physics, Sichuan University, Chengdu, Sichuan 610065, People’s Republic of China
}

Received: 12 May 2019 / Accepted: 28 June 2019 / Published online: 8 July 2019

(C) The Author(s) 2019

\begin{abstract}
We first use the Hamilton-Jacobi method to describe the motion in curved spacetime of a scalar particle and a fermion, which are shown to satisfy the same HamiltonJacobi equation. To investigate the laws of thermodynamics and the weak cosmic censorship, we focus on $D$-dimensional asymptotically AdS charged black hole solutions in general nonlinear electrodynamics (NLED). With absorbing a charged particle, the variation of the generic charged NLED black hole is calculated in the normal and extended phase spaces. In the normal phase space, where the cosmological constant and dimensionful parameters in NLED are fixed, the first and second laws of thermodynamics are satisfied. In the extended phase space, where the cosmological constant and dimensionful parameters in NLED are treated as thermodynamic variables, the first law of thermodynamics is also satisfied. However, the black hole entropy can either increase or decrease depending on the changes in the dimensionful parameters. Furthermore, we find that the weak cosmic censorship conjecture is valid for the extremal and near-extremal black holes in the both phase spaces.
\end{abstract}

\section{Contents}

1 Introduction . . . . . . . . . . . 1

2 Hamilton-Jacobi equation . . . . . . . . . . 2

2.1 Scalar particle . . . . . . . . . . . . 2

2.2 Fermion ................ 3

2.3 Motion near black hole horizon . . . . . . . . . 3

3 D-dimensional NLED black hole solution . . . . . . 4

4 Thermodynamics via charged particle absorption . . 5

4.1 Normal phase space . . . . . . . . . . . . . 6

\footnotetext{
a e-mail: pengw@scu.edu.cn

b e-mail: iverwu@scu.edu.cn

ce-mail: hyanga@scu.edu.cn
}

4.2 Extended phase space . . . . . . . . . . . . 6

5 Weak cosmic censorship conjecture . . . . . . 7

5.1 Normal phase space . . . . . . . . . . . . . . 7

5.2 Extended phase space . . . . . . . . . . . . 8

6 Conclusion . . . . . . . . . . . . 8

References . . . . . . . . . . . . . . . 9

\section{Introduction}

Explaining the thermodynamic nature of black holes can have a deep impact upon the understanding of quantum gravity. By making a black hole with an ergosphere absorb a particle with negative energy, Penrose noted that energy can be extracted from the black hole [1]. Later, an irreducible quantity, namely the irreducible mass, was found to exist when a black hole absorbs a particle [2-4]. Due to the similarity between the irreducible mass and the entropy, Bekenstein proposed that the area of a black hole (or the square of its irreducible mass) corresponds to its entropy [5,6]. Moreover, the four laws of black hole mechanics were established in [7], which are analogous to the laws of thermodynamics. Without using any complete quantum theory of gravity, the semi-classical approach allows for the computation of the thermodynamic quantities of black holes. Indeed, with the advent of quantum field theory in curved spacetime, Hawking found that black holes possess the temperature [8].

Investigating the thermodynamic properties of black holes has become quite a mature subject over the years. In particular, the study of asymptotically anti-deSitter black holes has become important since the discovery of the AdS/CFT correspondence [9]. Hawking and Page showed that a firstorder phase transition occurs between the Schwarzschild AdS black hole and the thermal AdS space [10], which was later understood as a confinement/deconfinement phase transition in the context of the AdS/CFT correspondence [11]. 
There has been much interest in studying the thermodynamics and phase structure of AdS black holes [12-16]. Recently, the asymptotically AdS black holes have also been studied in the context of the extended phase space thermodynamics, where the cosmological constant is interpreted as a thermodynamic pressure $[17,18]$. The $P-V$ criticality study and thermodynamics have been explored for various AdS black holes in the extended phase space [19-26], where some intriguing phase behavior, e.g., reentrant phase transitions, tricritical points, was found to be present. Therefore, studying the thermodynamics and phase structure of black holes in both the normal and extended phase spaces is important and interesting.

The weak cosmic censorship conjecture asserts that singularities are always hidden by event horizons in any real physical process from regular initial conditions and cannot be seen by the observers at the future null infinity [1]. To test the validity of the weak cosmic censorship conjecture, Wald attempted to overcharge or overspin an extremal KerrNewman black hole by throwing a test particle into the black hole [27]. However, for the particle with sufficient charge or angular momentum to overcharge or overspin the black hole, the black hole can not capture it because of the electromagnetic or centrifugal repulsion force. On the other hand, it was found that near-extremal charged/rotating black holes could be overcharged/overspun by absorbing a particle [2830]. However, subsequent researches showed that the weak cosmic censorship conjecture might be still valid for these black holes with consideration of the backreaction and selfforce effects [31-36]. Since there is still a lack of the general proof of the weak cosmic censorship conjecture, its validity has been tested in various black holes [37-60]. In particular, the thermodynamics and the weak cosmic censorship conjecture have been considered for a $D$-dimensional ReissnerNordstrom (RN)-AdS black via the charged particle absorption in the extended phase space [61]. It showed that the first law of thermodynamics and the weak cosmic censorship conjecture are satisfied while the second law of thermodynamics is violated near extremality. It is noteworthy that the second law of thermodynamics is always valid for a RN-AdS black hole in the normal phase space.

If quantum corrections are considered, nonlinear terms are usually added to the Maxwell Lagrangian, which is an effective model, namely the nonlinear electrodynamics (NLED). In the Einstein-NLED theory, various NLED charged black hole solutions were derived and discussed in a number of papers [62-71] (for a brief review see [72]). A globally regular NLED black hole solution with nonzero electric charge can be constructed, which requires that the NLED Lagrangian is strongly non-Maxwell in the weak field limit $[73,74]$. Specifically, the thermodynamics of NLED black holes has been considered in the literature, e.g., power Maxwell invariant black holes [75,76], non-linear magnetic- charged dS black holes [77], Born-Infeld AdS black holes [78-92], Born-Infeld black holes in a cavity [93]. The general $D$-dimensional, asymptotically flat or AdS, NLED charged black hole solutions were obtained in [94], where the black holes' structural and thermodynamic properties were carefully analyzed. In particular, the first law of black hole thermodynamics and the Smarr formula in the normal phase space were given there. However, to the best of our knowledge, the laws of thermodynamics in the extended phase space and the weak cosmic censorship conjecture have not been discussed in the context of a general $D$-dimensional NLED-AdS black hole.

In this paper, we extend the analysis of [61] (a RN-AdS black hole) to study the thermodynamics and the weak cosmic censorship conjecture for a $D$-dimensional AdS, NLED charged black hole in the normal and extended phase spaces. Our results are NLED model independent and show that the results of [61] are quite robust against the corrections from NLED. The rest of this paper is organized as follows. In Sect. 2, we derive the Hamilton-Jacobi equation for a particle in curved spacetime and discuss its motion around the black hole horizon. In Sect. 3, we review the $D$-dimensional NLED charged AdS black hole solution to lay the ground for studying the thermodynamics of the black hole and the weak cosmic censorship in the following sections. In Sect. 4, the thermodynamics of the black hole are discussed in the normal and extended phase spaces. We test the weak cosmic censorship conjecture in Sect. 5. We summarize our results in Sect. 6. For simplicity, we set $G=\hbar=c=k_{B}=1$ in this paper.

\section{Hamilton-Jacobi equation}

In [95], we have already derived the Hamilton-Jacobi equations for a scalar particle and a fermion in a curved spacetime background under an electric potential $A_{\mu}$ and showed that these Hamilton-Jacobi equations have the same form. Here, we first briefly review the derivation of the Hamilton-Jacobi equation. After the Hamilton-Jacobi equation is obtained, the motion of the particle near the horizon of a charged black hole is discussed. In this section, we temporarily restore $\hbar$ to keep the track of the leading term of the WKB expansion.

\subsection{Scalar particle}

In a semiclassical approximation, the Hamilton-Jacobi equation for a scalar particle is the lowest order of the WKB expansion of the corresponding Klein-Gordon equation. In curved spacetime, for a scalar particle of mass $m$ and charge $q$, the Klein-Gordon equation is 
$\left(\nabla^{\mu}-\frac{i q A^{\mu}}{\hbar}\right)\left(\nabla_{\mu}-\frac{i q A_{\mu}}{\hbar}\right) \phi+\frac{m^{2}}{\hbar^{2}} \phi=0$,

where $\nabla_{\mu}$ is the covariant derivative, and $A_{\mu}$ is the electromagnetic potential. Assuming the WKB ansatz for $\phi$ :

$\phi=A \exp \left(\frac{i I}{\hbar}\right)$

where $A$ is a slowly varying amplitude, one can expand Eq. (1) in powers of $\hbar$ and finds that the lowest order term is

$\left(\partial^{\mu} I-q A^{\mu}\right)\left(\partial_{\mu} I-q A_{\mu}\right)=m^{2}$.

which is the Hamilton-Jacobi equation.

\subsection{Fermion}

In curved spacetime, the Dirac equation for a spin-1/2 fermion of the mass $m$ and the charge $q$ takes on the form as

$i \gamma_{\mu}\left(\partial^{\mu}+\Omega^{\mu}-\frac{i q A^{\mu}}{\hbar}\right) \psi-\frac{m}{\hbar} \psi=0$,

where $\Omega_{\mu} \equiv \frac{i}{2} \omega_{\mu}^{a b} \Sigma_{a b}, \Sigma_{a b}$ is the Lorentz spinor generator, $\omega_{\mu}^{a b}$ is the spin connection and $\left\{\gamma_{\mu}, \gamma_{\nu}\right\}=2 g_{\mu \nu}$. The Greek indices are raised and lowered by the curved metric $g_{\mu \nu}$, while the Latin indices are governed by the flat metric $\eta_{a b}$. To obtain the Hamilton-Jacobi equation for the fermion, the WKB ansatz for $\psi$ is assumed as

$\psi=\exp \left(\frac{i I}{\hbar}\right) v$

where $v$ is a slowly varying spinor amplitude. Substituting Eq. (5) into Eq. (4), we find that the lowest order term of $\hbar$ is

$\gamma_{\mu}\left(\partial^{\mu} I-q A^{\mu}\right) v=-m v$

which is the Hamilton-Jacobi equation for the fermion. Multiplying both sides of Eq. (6) from the left by $\gamma_{v}\left(\partial^{v} I+e A^{v}\right)$ and then using Eq. (6) to simplify the RHS, one obtains

$\gamma_{v}\left(\partial^{v} I-q A^{v}\right) \gamma_{\mu}\left(\partial^{\mu} I-q A^{\mu}\right) v=m^{2} v$.

Using $\left\{\gamma_{\mu}, \gamma_{\nu}\right\}=2 g_{\mu \nu}$, we have

$\left[\left(\partial^{\mu} I-q A^{\mu}\right)\left(\partial_{\mu} I-q A_{\mu}\right)-m^{2}\right] v=0$.

Since $v$ is nonzero, the Hamilton-Jacobi equation reduces to

$\left(\partial^{\mu} I-q A^{\mu}\right)\left(\partial_{\mu} I-q A_{\mu}\right)=m^{2}$, which is the same as the Hamilton-Jacobi equation for a scalar.

\subsection{Motion near black hole horizon}

To include a broader class, we consider a $D$-dimensional static black hole with the line element

$d s^{2}=-f(r) d t^{2}+\frac{1}{f(r)} d r^{2}+C\left(r^{2}\right) h_{a b}(x) d x^{a} d x^{b}$,

where $a, b=1, \ldots D-2$. We also assume that there is a presence of electromagnetic potential $A_{\mu}$ with the following ansatz

$A_{\mu}=A_{t}(r) \delta_{\mu t}$

which means that the black hole is electrically charged. Suppose that the outermost horizon of the black hole is at $r=r_{+}$, which gives that $f\left(r_{+}\right)=0$ and $f(r)>0$ for $r>r_{+}$.

In the metric (10), the Hamilton-Jacobi equation (3) reduces to

$-\frac{\left(\partial_{t} I-q A_{t}\right)^{2}}{f(r)}+f(r)\left(\partial_{r} I\right)^{2}+\frac{h^{a b}(x) \partial_{a} I \partial_{b} I}{C\left(r^{2}\right)}=m^{2}$,

where $h^{a b} h_{b c}=\delta_{c}^{a}$. Since $I$ is the classical action, $E=$ $-\partial_{t} I$ is the conserved energy of the particle. To solve the Hamilton-Jacobi equation, we can employ the following ansatz

$I=-E t+W(r)+\Theta(x)$.

The method of separation of variables gives the differential equation for $\Theta(x)$

$h^{a b}(x) \partial_{a} \Theta(x) \partial_{b} \Theta(x)=\lambda$,

where $\lambda$ is a constant. On the other hand, the differential equation for $W(r)$ can lead to

$E=-q A_{t}(r)+\sqrt{f(r)\left[m^{2}+\frac{\lambda}{C\left(r^{2}\right)}\right]+\left[P^{r}(r)\right]^{2}}$,

where $P^{r}(r) \equiv f(r) \partial_{r} W(r)$ is the radial momentum of the particle, and we choose the positive sign in front of the square root since the energy of the particle is required to be a positive value [2,4]. As noted in [96], $P^{r}(r)$ is finite and nonzero at $r=r_{+}$, which accounts for the Hawing radiation 
modeled as a tunneling process. So very close to the outer horizon at $r=r_{+}$, Eq. (15) becomes

$E=q \Phi+\left|P^{r}\left(r_{+}\right)\right|$,

where $\Phi \equiv-A_{t}\left(r_{+}\right)$is the electric potential of the black hole. Equation (16) relates the energy of the particle to the momentum and the potential energy near $r=r_{+}$.

\section{D-dimensional NLED black hole solution}

For general NLED theories, the four and higher-dimensional static and spherically symmetric black hole solutions with an electric field have already been given in $[97,98]$ and [94], respectively. Here, we review the derivation of the $D$ dimensional NLED charged black hole solution to introduce the black hole setup for the rest of the paper. Consider a $D$-dimensional model of gravity coupled to a nonlinear electromagnetic field $A_{\mu}$ with the action given by

$S_{\text {Bulk }}=\int d^{D} x \sqrt{-g}\left[\frac{R-2 \Lambda}{16 \pi}+\frac{\mathcal{L}\left(s ; a_{i}\right)}{4 \pi}\right]$,

where the cosmological constant $\Lambda=-\frac{(D-1)(D-2)}{2 l^{2}}$, and $l$ is the AdS radius. In the action (17), we assume that the generic NLED Lagrangian $\mathcal{L}\left(s ; a_{i}\right)$ is a function of $s$ and the dimensionful parameters $a_{i}$. The parameters $a_{i}$ characterize the effects of nonlinearity in the NLED, and $s$ is an independent nontrivial scalar using $F_{\mu \nu}=\partial_{\mu} A_{\nu}-\partial_{\nu} A_{\mu}$ and none of its derivatives:

$s=-\frac{1}{4} F^{\mu v} F_{\mu \nu}$.

Varying the action (17) with respect to $g_{\mu \nu}$ and $A_{\mu}$, we find that the equations of motion are

$$
\begin{aligned}
R_{\mu \nu}-\frac{1}{2} R g_{\mu \nu}-\frac{(D-1)(D-2)}{2 l^{2}} g_{\mu \nu} & =8 \pi T_{\mu \nu}, \\
\nabla_{\mu} G^{\mu \nu} & =0
\end{aligned}
$$

where $T_{\mu \nu}$ is the energy-momentum tensor:

$$
T_{\mu \nu}=\frac{1}{4 \pi}\left[g_{\mu \nu} \mathcal{L}\left(s ; a_{i}\right)+\frac{\partial \mathcal{L}\left(s ; a_{i}\right)}{\partial s} F_{\mu}^{\rho} F_{\nu \rho}\right] .
$$

Here, we define the auxiliary fields $G^{\mu \nu}$ for later convenience:

$G^{\mu \nu} \equiv-\frac{\partial \mathcal{L}\left(s ; a_{i}\right)}{\partial F_{\mu \nu}}=\frac{\partial \mathcal{L}\left(s ; a_{i}\right)}{\partial s} F^{\mu \nu}$.
To construct a charged AdS black hole solution, we take the following ansatz for the metric and the NLED field

$$
\begin{aligned}
d s^{2} & =-f(r) d t^{2}+\frac{d r^{2}}{f(r)}+r^{2} d \Omega_{D-2}^{2}, \\
A_{\mu} d x^{\mu} & =A_{t}(r) d t
\end{aligned}
$$

where $d \Omega_{D-2}^{2}$ is the metric on the unit $(D-2)$-sphere. The equations of motion for $t t$-component of gravity and $A_{t}(r)$ are

$$
\begin{aligned}
& -\frac{(D-2)(D-3)}{2} \frac{f(r)\left[-1+f(r)-\frac{D-1}{D-3} \frac{r^{2}}{l^{2}}+\frac{r f^{\prime}(r)}{D-3}\right]}{r^{2}} \\
& \quad=-2 f(r)\left[\mathcal{L}\left(s ; a_{i}\right)+A_{t}^{\prime}(r) G^{r t}\right], \\
& {\left[r^{D-2} G^{t r}\right]^{\prime}=0,}
\end{aligned}
$$

respectively, where

$s=\frac{A_{t}^{\prime 2}(r)}{2}$ and $G^{t r}=\frac{\partial \mathcal{L}\left(s, a_{i}\right)}{\partial s} A_{t}^{\prime}(r)$.

It can show that the rest equations of motion can be derived from Eqs. (23) and (24). Solving Eq. (24) gives

$G^{t r}=\frac{\tilde{Q}}{r^{D-2}}$,

where $\tilde{Q}$ is a constant. From Eqs. (25) and (26), $A_{t}^{\prime}(r)$ is determined by

$\frac{\partial \mathcal{L}\left(A_{t}^{\prime 2}(r) / 2 ; a_{i}\right)}{\partial s} A_{t}^{\prime}(r)=\frac{\tilde{Q}}{r^{D-2}}$.

Furthermore, integrating Eq. (23) leads to

$$
\begin{aligned}
f(r)= & 1-\frac{\tilde{M}}{r^{D-3}}+\frac{r^{2}}{l^{2}}-\frac{4}{D-2} \frac{1}{r^{D-3}} \\
& \times \int_{r}^{\infty} r^{D-2}\left[\mathcal{L}\left(\frac{A_{t}^{\prime 2}(r)}{2} ; a_{i}\right)-A_{t}^{\prime}(r) \frac{\tilde{Q}}{r^{D-2}}\right] d r,
\end{aligned}
$$

where $\tilde{M}$ is a constant. For large values of $r$, one finds that

$$
f(r) \approx 1-\frac{\tilde{M}}{r^{D-3}}+\frac{r^{2}}{l^{2}}+\frac{2 \tilde{Q}^{2}}{(D-2)(D-3) r^{2(D-3)}},
$$

which reduces to the metric of a $D$-dimensional RN-AdS black hole. The Hawking temperature of the black hole is given by

$T=\frac{f^{\prime}\left(r_{+}\right)}{4 \pi}$. 
At $r=r_{+}$, Eq. (23) gives

$$
\begin{aligned}
T= & \frac{(D-3)}{4 \pi r_{+}}\left\{1+\frac{D-1}{D-3} \frac{r_{+}^{2}}{l^{2}}+\frac{4 r_{+}^{2}}{(D-2)(D-3)}\right. \\
& \left.\times\left[\mathcal{L}\left(\frac{A_{t}^{\prime 2}\left(r_{+}\right)}{2} ; a_{i}\right)-A_{t}^{\prime}\left(r_{+}\right) \frac{\tilde{Q}}{r_{+}^{D-2}}\right]\right\} .
\end{aligned}
$$

Since the black hole solution (28) is asymptotically AdS, the asymptotic behavior of $f(r)$ in Eq. (29) can relate the constant $\tilde{M}$ to the black hole mass $M$ [99],

$M=\frac{D-2}{16 \pi} \omega_{D-2} \tilde{M}$,

with $\omega_{D-2}$ being the volume of the unit $(D-2)$-sphere:

$\omega_{D-2}=\frac{2 \pi^{\frac{D-1}{2}}}{\Gamma\left(\frac{D-1}{2}\right)}$.

The black hole charge $Q$ can be expressed in terms of the constants $\tilde{Q}$. To do so, turning on an external current $J^{\mu}$ gives an interaction term:

$S_{I}=\int d^{D} x \sqrt{-g} J^{\mu} A_{\mu}$.

The equation of motion for $A_{\mu}$ then becomes

$\nabla_{\nu} G^{\mu \nu}=4 \pi J^{\mu}$

The charge passing through a spacelike $t$-constant hypersurface $\Sigma_{t}$ is given by

$Q=-\int_{\Sigma_{t}} J^{\mu} n_{\mu} d \Sigma$

where $n_{\mu}$ is the unit normal vector of $\Sigma_{t}$, and $d \Sigma$ is the surface element on $\Sigma_{t}$. Using Stokes' theorem, we rewrite $Q$ as an integral over the boundary of $\Sigma_{t}$, i.e., the $(D-2)$ sphere $S^{D-2}$ at $r=\infty$,

$Q=\frac{1}{8 \pi} \oint_{S^{D-2}} G^{\mu \nu} d S_{\mu \nu}=\frac{\tilde{Q}}{4 \pi} \omega_{D-2}$

where Eqs. (26) and (35) are used, and $d S_{\mu \nu}$ is the directed surface element on $S^{D-2}$. The electrical potential measured with respect to the horizon is

$\Phi=\int_{r_{+}}^{\infty} A_{t}^{\prime}(r)=-A_{t}\left(r_{+}\right)$

where we fix the field $A_{t}(r)$ at $r=\infty$ to be zero. The electrostatic potential $\Phi$ plays a role as the conjugated variable to $Q$ in black hole thermodynamics. The entropy of the black hole is one-quarter of the horizon area

$S=\frac{r_{+}^{D-2} \omega_{D-2}}{4}$

Besides $s$, there exist other invariants built from $F_{\mu \nu}$ using the totally antisymmetric Lorentz tensor $\epsilon^{\mu_{1} \cdots \mu_{D}}$. For example, in four-dimensional spacetime, one can have another invariant $p=-\frac{1}{8} \epsilon^{\mu \nu \rho \sigma} F_{\mu \nu} F_{\rho \sigma}$. The NLED Lagrangian can be a function of $s$ and $p$. In fact, Born-Infeld electrodynamics is described by the Lagrangian density

$\mathcal{L}_{\mathrm{BI}}(s, p ; a)=\frac{1}{a}\left(1-\sqrt{1-2 a s-a^{2} p^{2}}\right)$,

where the coupling parameter $a$ is related to the string tension $\alpha^{\prime}$ as $a=\left(2 \pi \alpha^{\prime}\right)^{2}$. However, when a black hole ansatz has no magnetic charge, the invariant $p$ always vanishes, and hence $\mathcal{L}_{\mathrm{BI}}(s, p ; a)$ and $\mathcal{L}_{\mathrm{BI}}(s, 0 ; a)$ would give the same solution. Therefore, focusing on black hole solutions that are not magnetically charged, our results also apply to the NLED with a more general Lagrangian containing not only $s$ but also other invariants that vanish for zero magnetic charge.

\section{Thermodynamics via charged particle absorption}

In this section, we investigate thermodynamics of the NLED black hole solution (22) by a charged particle entering the horizon. When the particle is absorbed by the black hole, the mass and charge of the black hole are varied due to the energy and charge conservation. Other thermodynamic variables (e.g., the entropy) would change accordingly. The aim of this section is to check whether the changes of the black hole thermodynamic variables satisfy the first and second law of thermodynamics in the normal and extended phase spaces. To do so, we first present some useful formulae:

$$
\begin{aligned}
& \left.\frac{\partial f(r)}{\partial r}\right|_{r=r_{+}}=4 \pi T, \\
& \left.\frac{\partial f(r)}{\partial M}\right|_{r=r_{+}}=-\frac{16 \pi}{(D-2) \omega_{D-2} r_{+}^{D-3}}, \\
& \left.\frac{\partial f(r)}{\partial l}\right|_{r=r+}=-\frac{2 r_{+}^{2}}{l^{3}}, \\
& \left.\frac{\partial f(r)}{\partial Q}\right|_{r=r_{+}}=\frac{16 \pi \Phi}{(D-2) \omega_{D-2} r_{+}^{D-3}}, \\
& \left.\frac{\partial f(r)}{\partial a_{i}}\right|_{r=r_{+}}=\frac{16 \pi \mathcal{A}_{i}}{(D-2) \omega_{D-2} r_{+}^{D-3}},
\end{aligned}
$$

where $\mathcal{A}_{i}$ is defined in Eq. (53), and Eq. (27) are used to obtain $\partial f(r) /\left.\partial Q\right|_{r=r_{+}}$and $\partial f(r) /\left.\partial a\right|_{r=r_{+}}$. 


\subsection{Normal phase space}

In the normal phase space, the AdS radius $l$ and the parameters $a_{i}$ are fixed and not treated as thermodynamic variables. So the black hole mass $M$ is the internal energy of the black hole. After the black hole absorbs a charged particle of the energy $E$ and charge $q$, the state of the black hole changes from $(M, Q)$ to $(M+d M, Q+d Q)$. The energy and charge conservation of the absorbing process gives

$d M=E \quad$ and $\quad d Q=q$,

where $E$ and $q$ are related via Eq. (16). To treat the particle as a test particle, we shall assume its energy $E$ and charge $q$ are small compared to those of the black hole,

$q=d Q \ll Q \quad$ and $\quad E=d M \ll M$.

For the initial state $(M, Q)$, the outer horizon radius of the black hole $r_{+}$satisfies

$f\left(r_{+} ; M, Q\right)=0$,

where the parameters $M$ and $Q$ are temporarily put explicitly as arguments of the function $f(r)$. When the black hole mass and charge are varied, the outer horizon radius moves to $r_{+}+$ $d r_{+}$, which also satisfies

$f\left(r_{+}+d r_{+} ; M+d M, Q+d Q\right)=0$.

So the infinitesimal changes in $M, Q$ and $r_{+}$are related by

$\left.\frac{\partial f(r)}{\partial r}\right|_{r=r_{+}} d r_{+}+\left.\frac{\partial f(r)}{\partial M}\right|_{r=r_{+}} d M+\left.\frac{\partial f(r)}{\partial Q}\right|_{r=r_{+}} d Q=0$.

Using Eqs. (16), (39) and (41), we find that the above equation reduces to

$\left|P^{r}\left(r_{+}\right)\right|=T d S$.

Therefore, the variation of entropy is

$d S=\frac{\left|P^{r}\left(r_{+}\right)\right|}{T}>0$,

which means the second law of thermodynamics is always satisfied. Substituting Eq. (47) into Eq. (16), we obtain

$d M=\Phi d Q+T d S$,

which is the first law of thermodynamics. Note that for the $D$-dimensional NLED charged black hole, the first law of thermodynamics in the normal phase space was already discussed in [94].

\subsection{Extended phase space}

Comparing with the first law of a usual thermodynamic system, one notes that there is an omission of a pressure volume term $P d V$ in Eq. (49). This observation motivates treating the cosmological constant as pressure associated with a black hole $[17,18]$ :

$P \equiv-\frac{\Lambda}{8 \pi}=\frac{(D-1)(D-2)}{16 \pi l^{2}}$.

In this case, the mass of the black hole is no longer identified with internal energy, rather it is regarded as a gravitational version of chemical enthalpy. So the conjugate thermodynamic volume of the black hole is given by

$V=\frac{\partial M}{\partial P}$.

Using Eq. (28) for $f(r)$ and $f\left(r_{+}\right)=0$, we find that the thermodynamic volume of the NLED black hole is

$V=\frac{\omega_{D-2}}{D-1} r_{+}^{D-1}$

To satisfy the Smarr relation, we need to further enlarge the phase space by promoting any dimensionful parameter in the theory to a thermodynamic variable $[19,100]$, which introduce the associated conjugate. The parameters and their conjugates add extra terms in the first law of thermodynamics. In particular, the parameter $a_{i}$ in the NLED theory (17) is considered as a thermodynamic phase space variable, and the associated conjugates $\mathcal{A}_{i}$ is

$\mathcal{A}_{i}=\frac{\partial M}{\partial a_{i}}=-\frac{\omega_{D-2}}{4 \pi} \int_{r_{+}}^{\infty} r^{D-2} \frac{\partial \mathcal{L}\left(s ; a_{i}\right)}{\partial a_{i}} d r$.

In [26], we showed that a complete Smarr relation is satisfied by all thermodynamic quantities in the extended phase space,

$M=2(T S-V P)+\sum_{i} c_{i} a_{i} \mathcal{A}_{i}+Q \Phi$,

where $\left[a_{i}\right]=L^{c_{i}}$.

After a charged particle of the energy $E$ and charge $q$ enters the black hole horizon, the internal energy and charge of the black hole are changed by

$d(M-P V)=E \quad$ and $\quad d Q=q$,

where $M-P V$ is the black hole internal energy in the extended phase space. Thus, the black hole changes from the initial state $\left(M, Q, l, a_{i}\right)$ to the final state $(M+d M, Q+$ 
$\left.d Q, l+d l, a_{i}+d a_{i}\right)$. The infinitesimal changes in $M, Q, l$, $a_{i}$ and $r_{+}$are related by

$$
\begin{gathered}
\left.\frac{\partial f(r)}{\partial r}\right|_{r=r_{+}} d r_{+}+\left.\frac{\partial f(r)}{\partial M}\right|_{r=r_{+}} d M+\left.\frac{\partial f(r)}{\partial Q}\right|_{r=r_{+}} d Q \\
+\left.\frac{\partial f(r)}{\partial l}\right|_{r=r_{+}} d l+\left.\frac{\partial f(r)}{\partial a_{i}}\right|_{r=r_{+}} d a_{i}=0 .
\end{gathered}
$$

From Eq. (56), we find that

$$
\left|P^{r}\left(r_{+}\right)\right|=T d S+\sum_{i} \mathcal{A}_{i} d a_{i}-P d V
$$

where we use Eq. (16) to express $d Q$ in terms of $\left|P^{r}\left(r_{+}\right)\right|$, and the derivatives of $f(r)$ are given in (41). Plugging the above equation into Eq. (16), we use Eq. (55) to obtain

$d M=\Phi d Q+T d S+V d P+\sum_{i} \mathcal{A}_{i} d a_{i}$,

which is the first law of thermodynamics.

Equation (57) gives the change of the black hole entropy,

$d S=\frac{\left|P^{r}\left(r_{+}\right)\right|-\sum_{i} \mathcal{A}_{i} d a_{i}}{T-\frac{(D-1) r_{+}}{4 \pi l^{2}}}$.

For large values of $T$, the denominator in Eq. (59) becomes

$$
T-\frac{(D-1) r_{+}}{4 \pi l^{2}} \sim \frac{(D-3)}{4 \pi r_{+}}>0,
$$

where we use Eq. (31). However for small enough $T$, the denominator is negative. Since $d a_{i}$ is arbitrary, the sign of the numerator in Eq. (59) is indefinite. In the extended phase space, the entropy can increases or decrease depending the values of $d a_{i}$. In the "restricted" extended phase space with $d a_{i}=0$, the change of the black hole entropy becomes

$d S=\frac{\left|P^{r}\left(r_{+}\right)\right|}{T-\frac{(D-1) r_{+}}{4 \pi l^{2}}}$,

which shows that the second law of thermodynamics is not satisfied for the extremal or near-extremal black hole. When the black hole is far enough from extremality, the second law is satisfied in the "restricted" extended phase space.

\section{Weak cosmic censorship conjecture}

To test the weak cosmic censorship conjecture, we make the black hole, which is initially extremal or near extremal, absorb a charged particle and check whether the resulting object has too much charge to be a black hole. Since the particle is a test particle, the black hole needs to start out close to extremal to have chance to become a naked singularity.
So here we assume that the initial NLED black hole is near extremal, for which there are two horizons. Between these two horizons, there exists one and only one minimum point at $r=r_{\min }$ for $f(r)$. Moreover, the minimum value of $f(r)$ is not greater than zero,

$\delta \equiv f\left(r_{\min }\right) \leq 0$,

and $\delta=0$ corresponds to the extremal black hole. After the black hole absorbs a charged particle, the minimum point would move to $r_{\min }+d r_{\min }$. For the final black hole solution, if the minimum value of $f(r)$ at $r=r_{\min }+d r_{\min }$ is still not greater than zero, there is an event horizon. Otherwise, the final black hole solution is over the extremal limit, and the weak cosmic censorship conjecture is violated. Again, we first present some useful formulae:

$$
\begin{aligned}
& \left.\frac{\partial f(r)}{\partial r}\right|_{r=r_{\text {min }}}=0, \\
& \left.\frac{\partial f(r)}{\partial M}\right|_{r=r_{\min }}=-\frac{16 \pi}{(D-2) \omega_{D-2} r_{\min }^{D-3}}, \\
& \left.\frac{\partial f(r)}{\partial l}\right|_{r=r_{\min }}=-\frac{2 r_{\min }^{2}}{l^{3}}, \\
& \left.\frac{\partial f(r)}{\partial Q}\right|_{r=r_{\min }}=\frac{16 \pi\left[\Phi+A_{t}\left(r_{+}\right)-A_{t}\left(r_{\min }\right)\right]}{(D-2) \omega_{D-2} r_{\min }^{D-3}}, \\
& \left.\frac{\partial f(r)}{\partial a_{i}}\right|_{r=r_{\min }}=\frac{16 \pi\left(\mathcal{A}_{i}+\delta \mathcal{A}_{i}\right)}{(D-2) \omega_{D-2} r_{\min }^{D-3}},
\end{aligned}
$$

where Eq. (27) are used to obtain $\partial f(r) /\left.\partial Q\right|_{r=r_{\min }}$ and $\partial f(r) /\left.\partial a_{i}\right|_{r=r_{\text {min }}}$, and we define

$\delta \mathcal{A}_{i} \equiv-\frac{\omega_{D-2}}{4 \pi} \int_{r_{\min }}^{r_{+}} d r r^{2} \frac{\partial \mathcal{L}\left(s ; a_{i}\right)}{\partial a_{i}}$

\subsection{Normal phase space}

In this case, absorbing a charged particle of the energy $E$ and charge $q$ makes the black hole change from the initial state $(M, Q)$ to the final state $(M+d M, Q+d Q)$, where $d M$ and $d Q$ are given in Eq. (42). Owing to these changes, the location of the minimum point of $f(r)$ moves to $r_{\min }+d r_{\min }$. For the final state, the minimum value of $f(r)$ at $r=r_{\min }+$ $d r_{\text {min }}$ becomes

$$
\begin{aligned}
f & \left(r_{\min }+d r_{\min } ; M+d M, Q+d Q\right) \\
& =\delta+\left.\frac{\partial f}{\partial r}\right|_{r=r_{\min }} d r_{\min }+\left.\frac{\partial f}{\partial M}\right|_{r=r_{\min }} d M+\left.\frac{\partial f}{\partial Q}\right|_{r=r_{\min }} d Q \\
& =\delta-\frac{16 \pi\left|P^{r}\left(r_{+}\right)\right|}{(D-2) \omega_{D-2} r_{\min }^{D-3}}+\frac{16 \pi q\left[A_{t}\left(r_{+}\right)-A_{t}\left(r_{\min }\right)\right]}{(D-2) \omega_{D-2} r_{\min }^{D-3}},
\end{aligned}
$$


where the parameters of $f(r)$ are put explicitly. If the initial black hole is extremal, we have $r_{+}=r_{\min }$ and $\delta=0$. The minimum value of $f(r)$ of the final black hole reduces to

$f\left(r_{\min }+d r_{\min }\right)=-\frac{16 \pi\left|P^{r}\left(r_{+}\right)\right|}{(D-2) \omega_{D-2} r_{\min }^{D-3}}<0$,

which implies that the extremal black hole becomes a nonextremal one by the absorption. For the near-extremal black hole, we define $\epsilon$ such that

$r_{\min }=r_{+}(1-\epsilon)$,

where $\epsilon \ll 1$. So $\delta$ is suppressed by $\epsilon$ in the near-extremal limit. Moreover, the second term in the third line of Eq. (65) is only suppressed by the test particle limit while the third term is suppressed by both near-extremal limit and the test particle limit. Therefore, in the test particle limit for the nearextremal black hole, the third term can be neglected, and Eq. (65) then gives

$f\left(r_{\min }+d r_{\min }\right)=\delta-\frac{16 \pi\left|P^{r}\left(r_{+}\right)\right|}{(D-2) \omega_{D-2} r_{\min }^{D-3}}<0$,

which means that the near-extremal black hole stays nonextremal after the absorption. In the normal phase space, the weak cosmic censorship conjecture is satisfied for the extremal and near-extremal NLED black holes under the charge particle absorption.

\subsection{Extended phase space}

In the extended phase space, after absorbing a charged particle, the parameters of the black hole change from $\left(M, Q, l, a_{i}\right)$ to $\left(M+d M, Q+d Q, l+d l, a_{i}+d a_{i}\right)$, and $r_{\text {min }}$ moves accordingly to $r_{\min }+d r_{\text {min }}$. The minimum value of $f(r)$ at $r=r_{\min }+d r_{\min }$ of the final state is given by

$$
\begin{aligned}
f( & \left.r_{\min }+d r_{\min } ; M+d M, Q+d Q, l+d l, a_{i}+d a_{i}\right) \\
= & \delta+\left.\frac{\partial f}{\partial M}\right|_{r=r_{\text {min }}} d M+\left.\frac{\partial f}{\partial Q}\right|_{r=r_{\text {min }}} d Q+\left.\frac{\partial f}{\partial l}\right|_{r=r_{\text {min }}} d l \\
& +\left.\sum_{i} \frac{\partial f}{\partial a_{i}}\right|_{r=r_{\text {min }}} d a_{i} \\
= & \delta-\frac{16 \pi\left\{T d S+\left[A_{t}\left(r_{+}\right)-A_{t}\left(r_{\min }\right)\right] q+\delta \mathcal{A}_{i} d a_{i}\right\}}{(D-2) \omega_{D-2} r_{\min }^{D-3}} \\
& +2\left(\frac{r_{+}^{D-1}}{r_{\text {min }}^{D-3}}-r_{\min }^{2}\right) \frac{d l}{l^{3}},
\end{aligned}
$$

where the parameters of $f(r)$ are put explicitly, and we use Eq. (63) for the derivatives of $f(r)$. When the initial black hole is extremal, one has that $r_{+}=r_{\min }, T=0, \delta \mathcal{A}_{i}=0$ and $\delta=0$. So the minimum value of $f(r)$ of the final black hole is

$f\left(r_{\min }+d r_{\min }\right)=0$,

which implies that the extremal black hole stays extremal after the absorption. For the near-extremal black hole in the test particle limit, the second and third terms in the third line of Eq. (69) are suppressed by both the near-extremal limit and the test particle limit and hence can be neglected. Therefore, Eq. (69) leads to

$f\left(r_{\min }+d r_{\min }\right)=\delta<0$,

which means that the near-extremal black hole stays nonextremal after the absorption. In the extended phase space, the weak cosmic censorship conjecture is also satisfied for the extremal and near-extremal NLED black holes under the charge particle absorption.

\section{Conclusion}

In this paper, we calculated the variations of the thermodynamic quantities of a general NLED-AdS black hole via

Table 1 Results for the first and second laws of thermodynamics and the weak cosmic censorship conjectures (WCCC), which are tested for a general NLED charged AdS black hole with absorbing a charged particle in the test particle limit

\begin{tabular}{lll}
\hline & Normal phase space & Extended phase space \\
\hline $\begin{array}{l}\text { 1st law } \\
\text { 2nd law }\end{array}$ & $\begin{array}{l}\text { Satisfied } \\
\text { Satisfied }\end{array}$ & $\begin{array}{l}\text { Satisfied } \\
\text { Indefinite. If } d a_{i}=0 \text { is imposed, the 2nd law is violated for the } \\
\text { extremal and near-extremal black holes }\end{array}$ \\
WCCC & $\begin{array}{l}\text { Satisfied for the extremal and near-extremal black } \\
\text { holes. After the charge particle absorption, the } \\
\text { extremal black hole becomes non-extremal. }\end{array}$ & $\begin{array}{l}\text { Satisfied for the extremal and near-extremal black holes. After } \\
\text { the charge particle absorption, the extremal black hole stays } \\
\text { extremal }\end{array}$ \\
\hline
\end{tabular}


absorbing a charged practice. With these variations, we checked the first and second laws of thermodynamics and the weak cosmic censorship conjecture for the NLED black hole in the test particle limit. Two scenarios were considered, namely the normal phase space and the extended phase space. Our results are summarized in Table 1.

In [61], the first and second laws of thermodynamics and the weak cosmic censorship conjecture have been tested for the Einstein-Maxwell action in the $D$-dimensional spacetime, which is the special case of the Einstein-NLED theory, i.e., $\mathcal{L}\left(s ; a_{i}\right)=s$. The analysis was considered in the extended phase space, in which there is no dimensionful parameter in the Maxwell Lagrangian. As expected, our results successfully reproduced those in [61]. Moreover, our results show that the correction terms to the electromagnetic part can not solve the violation of the second law of thermodynamics near extremality, and hence the correction terms to the gravity part should be considered. In [56], the thermodynamics and the weak cosmic censorship conjecture of the Born-Infeld AdS black holes were discussed in the normal and extended phase space. Our results agree with those in the normal phase space. However, in the extended space, it showed there that the extremal black holes will change into non-extremal black holes after the charge particle absorption, which can not reduce to the Maxwell case in [61] with $b=0$. Recently, a $(2+1)$-dimensional regular black hole with some nonlinear electrodynamic source was considered in [101], the results of which agree with ours.

Acknowledgements This work is supported in part by NSFC (Grant No. 11875196, 11375121 and 11005016).

Data Availability Statement This manuscript has no associated data or the data will not be deposited. [Authors' comment: The results of this paper are based on the theoretical derivation. We do not use any data in the calculations.]

Open Access This article is distributed under the terms of the Creative Commons Attribution 4.0 International License (http://creativecomm ons.org/licenses/by/4.0/), which permits unrestricted use, distribution, and reproduction in any medium, provided you give appropriate credit to the original author(s) and the source, provide a link to the Creative Commons license, and indicate if changes were made. Funded by SCOAP ${ }^{3}$.

\section{References}

1. R. Penrose, Gravitational collapse: the role of general relativity. Riv. Nuovo Cim. 1, 252 (1969) [Gen. Relativ. Gravit. 34, 1141 (2002)]

2. D. Christodoulou, Reversible and irreversible transforations in black hole physics. Phys. Rev. Lett. 25, 1596 (1970). https://doi. org/10.1103/PhysRevLett.25.1596

3. J.M. Bardeen, Kerr metric black holes. Nature 226, 64 (1970). https://doi.org/10.1038/226064a0
4. D. Christodoulou, R. Ruffini, Reversible transformations of a charged black hole. Phys. Rev. D 4, 3552 (1971). https://doi.org/ 10.1103/PhysRevD.4.3552

5. J.D. Bekenstein, Black holes and the second law. Lett. Nuovo Cim. 4, 737 (1972). https://doi.org/10.1007/BF02757029

6. J.D. Bekenstein, Black holes and entropy. Phys. Rev. D 7, 2333 (1973). https://doi.org/10.1103/PhysRevD.7.2333

7. J.M. Bardeen, B. Carter, S.W. Hawking, The four laws of black hole mechanics. Commun. Math. Phys. 31, 161 (1973). https:// doi.org/10.1007/BF01645742

8. S.W. Hawking, Particle creation by black holes. Commun. Math. Phys. 43, 199 (1975) Erratum: [Commun. Math. Phys. 46, 206 (1976)]. https://doi.org/10.1007/BF02345020. https://doi.org/10. 1007/BF01608497

9. J.M. Maldacena, The large $\mathrm{N}$ limit of superconformal field theories and supergravity. Int. J. Theor. Phys. 38, 1113 (1999) [Adv. Theor. Math. Phys. 2, 231 (1998)]. https://doi. org/10.1023/A:1026654312961. https://doi.org/10.4310/ATMP. 1998.v2.n2.a1. arXiv:hep-th/9711200

10. S.W. Hawking, D.N. Page, Thermodynamics of black holes in anti-De Sitter space. Commun. Math. Phys. 87, 577 (1983). https://doi.org/10.1007/BF01208266

11. E. Witten, Anti-de Sitter space, thermal phase transition, and confinement in gauge theories. Adv. Theor. Math. Phys. 2, 505 (1998). https://doi.org/10.4310/ATMP.1998.v2.n3.a3. arXiv:hep-th/9803131

12. A. Chamblin, R. Emparan, C.V. Johnson, R.C. Myers, Charged AdS black holes and catastrophic holography. Phys. Rev. D 60, 064018 (1999). https://doi.org/10.1103/PhysRevD.60.064018. arXiv:hep-th/9902170

13. A. Chamblin, R. Emparan, C.V. Johnson, R.C. Myers, Holography, thermodynamics and fluctuations of charged AdS black holes. Phys. Rev. D 60, 104026 (1999). https://doi.org/10.1103/ PhysRevD.60.104026. arXiv:hep-th/9904197

14. M.M. Caldarelli, G. Cognola, D. Klemm, Thermodynamics of Kerr-Newman-AdS black holes and conformal field theories. Class. Quantum Gravity 17, 399 (2000). https://doi.org/10.1088/ 0264-9381/17/2/310. arXiv:hep-th/9908022

15. R.G. Cai, Gauss-Bonnet black holes in AdS spaces. Phys. Rev. D 65, 084014 (2002). https://doi.org/10.1103/PhysRevD.65. 084014. arXiv:hep-th/0109133

16. D. Kubiznak, R.B. Mann, P-V criticality of charged AdS black holes. JHEP 1207, 033 (2012). https://doi.org/10.1007/ JHEP07(2012)033. arXiv:1205.0559 [hep-th]

17. D. Kastor, S. Ray, J. Traschen, Enthalpy and the mechanics of AdS black holes. Class. Quantum Gravity 26, 195011 (2009). https:// doi.org/10.1088/0264-9381/26/19/195011. arXiv:0904.2765 [hep-th]

18. B.P. Dolan, Pressure and volume in the first law of black hole thermodynamics. Class. Quantum Gravity 28, 235017 (2011). https://doi.org/10.1088/0264-9381/28/23/235017. arXiv:1106.6260 [gr-qc]

19. S. Gunasekaran, R.B. Mann, D. Kubiznak, Extended phase space thermodynamics for charged and rotating black holes and BornInfeld vacuum polarization. JHEP 1211, 110 (2012). https://doi. org/10.1007/JHEP11(2012)110. arXiv:1208.6251 [hep-th]

20. S.W. Wei, Y.X. Liu, Critical phenomena and thermodynamic geometry of charged Gauss-Bonnet AdS black holes. Phys. Rev. D 87(4), 044014 (2013). https://doi.org/10.1103/PhysRevD.87. 044014. arXiv:1209.1707 [gr-qc]

21. R.G. Cai, L.M. Cao, L. Li, R.Q. Yang, P-V criticality in the extended phase space of Gauss-Bonnet black holes in AdS space. JHEP 1309, 005 (2013). https://doi.org/10.1007/ JHEP09(2013)005. arXiv:1306.6233 [gr-qc]

22. W. Xu, L. Zhao, Critical phenomena of static charged AdS black holes in conformal gravity. Phys. Lett. B 736, 214 (2014). https:// 
doi.org/10.1016/j.physletb.2014.07.019. arXiv:1405.7665 [grqc]

23. A.M. Frassino, D. Kubiznak, R.B. Mann, F. Simovic, Multiple reentrant phase transitions and triple points in lovelock thermodynamics. JHEP 1409, 080 (2014). https://doi.org/10.1007/ JHEP09(2014)080. arXiv:1406.7015 [hep-th]

24. M.H. Dehghani, S. Kamrani, A. Sheykhi, $P-V$ criticality of charged dilatonic black holes. Phys. Rev. D 90(10), 104020 (2014). https://doi.org/10.1103/PhysRevD.90.104020. arXiv: 1505.02386 [hep-th]

25. R.A. Hennigar, W.G. Brenna, R.B. Mann, $P-V$ criticality in quasitopological gravity. JHEP 1507, 077 (2015). https://doi.org/ 10.1007/JHEP07(2015)077. arXiv:1505.05517 [hep-th]

26. P. Wang, H. Wu, H. Yang, Thermodynamics and phase transitions of nonlinear electrodynamics black holes in an extended phase space. arXiv: 1808.04506 [gr-qc]

27. R. Wald, Gedanken experiments to destroy a black hole. Ann. Phys. 82, 548 (1974). https://doi.org/10.1016/ 0003-4916(74)90125-0

28. V.E. Hubeny, Overcharging a black hole and cosmic censorship. Phys. Rev. D 59, 064013 (1999). https://doi.org/10.1103/ PhysRevD.59.064013. arXiv:gr-qc/9808043

29. T. Jacobson, T.P. Sotiriou, Over-spinning a black hole with a test body. Phys. Rev. Lett. 103, 141101 (2009). Erratum: [Phys. Rev. Lett. 103, 209903 (2009)]. https://doi.org/10.1103/ PhysRevLett.103.209903. https://doi.org/10.1103/PhysRevLett. 103.141101. [arXiv:0907.4146 [gr-qc]]

30. A. Saa, R. Santarelli, Destroying a near-extremal Kerr-Newman black hole. Phys. Rev. D 84, 027501 (2011). https://doi.org/10. 1103/PhysRevD.84.027501. arXiv:1105.3950 [gr-qc]

31. S. Hod, Weak cosmic censorship: as strong as ever. Phys. Rev. Lett. 100, 121101 (2008). https://doi.org/10.1103/PhysRevLett. 100.121101. arXiv:0805.3873 [gr-qc]

32. E. Barausse, V. Cardoso, G. Khanna, Test bodies and naked singularities: is the self-force the cosmic censor? Phys. Rev. Lett. 105, 261102 (2010). https://doi.org/10.1103/PhysRevLett.105. 261102. arXiv:1008.5159 [gr-qc]

33. E. Barausse, V. Cardoso, G. Khanna, Testing the cosmic censorship conjecture with point particles: the effect of radiation reaction and the self-force. Phys. Rev. D 84, 104006 (2011). https://doi. org/10.1103/PhysRevD.84.104006. arXiv:1106.1692 [gr-qc]

34. P.Zimmerman, I. Vega, E. Poisson, R. Haas, Self-force as a cosmic censor. Phys. Rev. D 87(4), 041501 (2013). https://doi.org/10. 1103/PhysRevD.87.041501. arXiv:1211.3889 [gr-qc]

35. M. Colleoni, L. Barack, Overspinning a Kerr black hole: the effect of self-force. Phys. Rev. D 91, 104024 (2015). https://doi.org/10. 1103/PhysRevD.91.104024. arXiv:1501.07330 [gr-qc]

36. M. Colleoni, L. Barack, A.G. Shah, M. van de Meent, Self-force as a cosmic censor in the Kerr overspinning problem. Phys. Rev. D 92(8), 084044 (2015). https://doi.org/10.1103/PhysRevD.92. 084044. arXiv:1508.04031 [gr-qc]

37. G.E.A. Matsas, A.R.R. da Silva, Overspinning a nearly extreme charged black hole via a quantum tunneling process. Phys. Rev. Lett. 99, 181301 (2007). https://doi.org/10.1103/PhysRevLett.99. 181301. arXiv:0706.3198 [gr-qc]

38. M. Richartz, A. Saa, Overspinning a nearly extreme black hole and the weak cosmic censorship conjecture. Phys. Rev. D 78, 081503 (2008). https://doi.org/10.1103/PhysRevD.78. 081503. arXiv:0804.3921 [gr-qc]

39. S. Isoyama, N. Sago, T. Tanaka, Cosmic censorship in overcharging a Reissner-Nordstróm black hole via charged particle absorption. Phys. Rev. D 84, 124024 (2011). https://doi.org/10.1103/ PhysRevD.84.124024. arXiv:1108.6207 [gr-qc]

40. S. Gao, Y. Zhang, Destroying extremal Kerr-Newman black holes with test particles. Phys. Rev. D 87(4), 044028 (2013). https://doi. org/10.1103/PhysRevD.87.044028. arXiv:1211.2631 [gr-qc]
41. S. Hod, Cosmic censorship: formation of a shielding horizon around a fragile horizon. Phys. Rev. D 87(2), 024037 (2013). https://doi.org/10.1103/PhysRevD.87.024037. arXiv:1302.6658 [gr-qc]

42. K. Duztas, I. Semiz, Cosmic censorship, black holes and integerspin test fields. Phys. Rev. D 88(6), 064043 (2013). https://doi. org/10.1103/PhysRevD.88.064043. arXiv:1307.1481 [gr-qc]

43. H.M. Siahaan, Destroying Kerr-Sen black holes. Phys. Rev. D 93(6), 064028 (2016). https://doi.org/10.1103/PhysRevD.93. 064028. arXiv:1512.01654 [gr-qc]

44. J. Natario, L. Queimada, R. Vicente, Test fields cannot destroy extremal black holes. Class. Quantum Gravity 33(17), 175002 (2016). https://doi.org/10.1088/0264-9381/33/ 17/175002. arXiv:1601.06809 [gr-qc]

45. K. Duztas, Overspinning BTZ black holes with test particles and fields. Phys. Rev. D 94(12), 124031 (2016). https://doi.org/10. 1103/PhysRevD.94.124031. arXiv:1701.07241 [gr-qc]

46. K.S. Revelar, I. Vega, Overcharging higher-dimensional black holes with point particles. Phys. Rev. D 96(6), 064010 (2017). https://doi.org/10.1103/PhysRevD.96.064010. arXiv: 1706.07190 [gr-qc]

47. J. Sorce, R.M. Wald, Gedanken experiments to destroy a black hole. II. Kerr-Newman black holes cannot be overcharged or overspun. Phys. Rev. D 96(10), 104014 (2017). https://doi.org/ 10.1103/PhysRevD.96.104014. arXiv:1707.05862 [gr-qc]

48. V. Husain, S. Singh, Penrose inequality in anti-de Sitter space. Phys. Rev. D 96(10), 104055 (2017). https://doi.org/10.1103/ PhysRevD.96.104055. arXiv:1709.02395 [gr-qc]

49. T. Crisford, G.T. Horowitz, J.E. Santos, Testing the weak gravity-cosmic censorship connection. Phys. Rev. D 97(6), 066005 (2018). https://doi.org/10.1103/PhysRevD.97.066005. arXiv:1709.07880 [hep-th]

50. J. An, J. Shan, H. Zhang, S. Zhao, Five-dimensional MyersPerry black holes cannot be overspun in gedanken experiments. Phys. Rev. D 97(10), 104007 (2018). https://doi.org/10.1103/ PhysRevD.97.104007. arXiv:1711.04310 [hep-th]

51. B. Ge, Y. Mo, S. Zhao, J. Zheng, Higher-dimensional charged black holes cannot be over-charged by gedanken experiments. Phys. Lett. B 783, 440 (2018). https://doi.org/10.1016/j.physletb. 2018.07.015. arXiv:1712.07342 [hep-th]

52. T.Y. Yu, W.Y. Wen, Cosmic censorship and weak gravity conjecture in the Einstein-Maxwell-dilaton theory. Phys. Lett. B 781, 713 (2018). https://doi.org/10.1016/j.physletb.2018.04.060. arXiv: 1803.07916 [gr-qc]

53. B. Gwak, Weak cosmic censorship conjecture in Kerr-(Anti-)de Sitter black hole with scalar field. JHEP 1809, 081 (2018). https:// doi.org/10.1007/JHEP09(2018)081. arXiv:1807.10630 [gr-qc]

54. Y. Gim, B. Gwak, Charged black hole in gravity's rainbow: violation of weak cosmic censorship. arXiv:1808.05943 [gr-qc]

55. D. Chen, Weak cosmic censorship conjecture in BTZ black holes with scalar fields. arXiv: 1812.03459 [gr-qc]

56. X.X. Zeng, H.Q. Zhang, Thermodynamics and weak cosmic censorship conjecture in Born-Infeld-anti-de Sitter black holes. arXiv:1901.04247 [hep-th]

57. D. Chen, X. Zeng, W. Yang, Thermodynamics and weak cosmic censorship conjecture in Reissner-Nordström anti-de Sitter black holes with scalar field. arXiv:1901.05140 [hep-th]

58. B. Gwak, Weak cosmic censorship with pressure and volume in charged Anti-de Sitter black hole under charged scalar field. arXiv:1901.05589 [gr-qc]

59. X.X.Zeng, Y.W. Han, D.Y. Chen, Thermodynamics and weak cosmic censorship conjecture of the BTZ black holes in the extended phase space. arXiv:1901.08915 [gr-qc]

60. D. Chen, Thermodynamics and weak cosmic censorship conjecture in extended phase spaces of anti-de Sitter black holes with particles' absorption. arXiv:1902.06489 [hep-th] 
61. B. Gwak, Thermodynamics with pressure and volume under charged particle absorption. JHEP 1711, 129 (2017). https://doi. org/10.1007/JHEP11(2017)129. arXiv:1709.08665 [gr-qc]

62. H.H. Soleng, Charged black points in general relativity coupled to the logarithmic U(1) gauge theory. Phys. Rev. D 52, 6178 (1995). https://doi.org/10.1103/PhysRevD.52.6178. arXiv:hep-th/9509033

63. E. Ayon-Beato, A. Garcia, Regular black hole in general relativity coupled to nonlinear electrodynamics. Phys. Rev. Lett. 80, 5056 (1998). https://doi.org/10.1103/PhysRevLett.80.5056. arXiv:gr-qc/9911046

64. H. Maeda, M. Hassaine, C. Martinez, Lovelock black holes with a nonlinear Maxwell field. Phys. Rev. D 79, 044012 (2009). https:// doi.org/10.1103/PhysRevD.79.044012. arXiv:0812.2038 [gr-qc]

65. Z.Y. Fan, X. Wang, Construction of regular black holes in general relativity. Phys. Rev. D 94(12), 124027 (2016). https://doi.org/10. 1103/PhysRevD.94.124027. arXiv:1610.02636 [gr-qc]

66. Z.Y. Fan, Critical phenomena of regular black holes in anti-de Sitter space-time. Eur. Phys. J. C 77(4), 266 (2017). https://doi.org/ 10.1140/epjc/s10052-017-4830-9. arXiv:1609.04489 [hep-th]

67. S .H. Hendi, B. Eslam Panah, S. Panahiyan, A. Sheykhi, Dilatonic BTZ black holes with power-law field. Phys. Lett. B 767, 214 (2017). https://doi.org/10.1016/j.physletb.2017.01.066. arXiv: 1703.03403 [gr-qc]

68. J. Tao, P. Wang, H. Yang, Testing holographic conjectures of complexity with Born-Infeld black holes. Eur. Phys. J. C 77(12), 817 (2017). https://doi.org/10.1140/epjc/s10052-017-5395-3. arXiv: 1703.06297 [hep-th]

69. X. Guo, P. Wang, H. Yang, Membrane paradigm and holographic DC conductivity for nonlinear electrodynamics. Phys. Rev. D 98(2), 026021 (2018). https://doi.org/10.1103/PhysRevD. 98.026021. arXiv:1711.03298 [hep-th]

70. B. Mu, P. Wang, H. Yang, Holographic DC conductivity for a power-law Maxwell field. Eur. Phys. J. C 78(12), 1005 (2018). https://doi.org/10.1140/epjc/s10052-018-6491-8. arXiv: 1711.06569 [hep-th]

71. X.M. Kuang, B. Liu, A. Ovgun, Nonlinear electrodynamics AdS black hole and related phenomena in the extended thermodynamics. Eur. Phys. J. C 78(10), 840 (2018). https://doi.org/10.1140/ epjc/s10052-018-6320-0. arXiv:1807.10447 [gr-qc]

72. K.A. Bronnikov, Dyonic configurations in nonlinear electrodynamics coupled to general relativity. Grav. Cosmol. 23(4), 343 (2017). https://doi.org/10.1134/S0202289317040053. arXiv: 1708.08125 [gr-qc]

73. K.A. Bronnikov, Regular magnetic black holes and monopoles from nonlinear electrodynamics. Phys. Rev. D 63, 044005 (2001). https://doi.org/10.1103/PhysRevD.63.044005. arXiv:gr-qc/0006014

74. K.A. Bronnikov, Comment on 'Regular black hole in general relativity coupled to nonlinear electrodynamics'. Phys. Rev. Lett. 85, 4641 (2000). https://doi.org/10.1103/PhysRevLett.85.4641

75. S.H. Hendi, M.H. Vahidinia, Extended phase space thermodynamics and $\mathrm{P}-\mathrm{V}$ criticality of black holes with a nonlinear source. Phys. Rev. D 88(8), 084045 (2013). https://doi.org/10. 1103/PhysRevD.88.084045. arXiv:1212.6128 [hep-th]

76. J.X. Mo, G.Q. Li, X.B. Xu, Effects of power-law Maxwell field on the critical phenomena of higher dimensional dilaton black holes. Phys. Rev. D 93(8), 084041 (2016). https://doi.org/10. 1103/PhysRevD.93.084041. arXiv:1601.05500 [gr-qc]

77. C.H. Nam, Non-linear charged dS black hole and its thermodynamics and phase transitions. Eur. Phys. J. C 78(5), 418 (2018). https://doi.org/10.1140/epjc/s10052-018-5922-x

78. S. Fernando, D. Krug, Charged black hole solutions in Einstein-Born-Infeld gravity with a cosmological constant. Gen. Relativ. Gravit. 35, 129 (2003). https://doi.org/10.1023/A: 1021315214180. arXiv:hep-th/0306120
79. S. Fernando, Thermodynamics of Born-Infeld-anti-de Sitter black holes in the grand canonical ensemble. Phys. Rev. D 74, 104032 (2006). https://doi.org/10.1103/PhysRevD.74.104032. arXiv:hep-th/0608040

80. R. Banerjee, S. Ghosh, D. Roychowdhury, New type of phase transition in Reissner-Nordstrom-AdS black hole and its thermodynamic geometry. Phys. Lett. B 696, 156 (2011). https://doi.org/ 10.1016/j.physletb.2010.12.010. arXiv:1008.2644 [gr-qc]

81. R. Banerjee, D. Roychowdhury, Critical phenomena in BornInfeld AdS black holes. Phys. Rev. D 85, 044040 (2012). https:// doi.org/10.1103/PhysRevD.85.044040. arXiv:1111.0147 [gr-qc]

82. A. Lala, D. Roychowdhury, Ehrenfest's scheme and thermodynamic geometry in Born-Infeld AdS black holes. Phys. Rev. D 86, 084027 (2012). https://doi.org/10.1103/PhysRevD.86. 084027. arXiv:1111.5991 [gr-qc]

83. R. Banerjee, D. Roychowdhury, Critical behavior of BornInfeld AdS black holes in higher dimensions. Phys. Rev. D 85, 104043 (2012). https://doi.org/10.1103/PhysRevD.85.104043. arXiv:1203.0118 [gr-qc]

84. D.C. Zou, S.J. Zhang, B. Wang, Critical behavior of Born-Infeld AdS black holes in the extended phase space thermodynamics. Phys. Rev. D 89(4), 044002 (2014). https://doi.org/10.1103/ PhysRevD.89.044002. arXiv:1311.7299 [hep-th]

85. M. Azreg-Ainou, Black hole thermodynamics: no inconsistency via the inclusion of the missing $P-V$ terms. Phys. Rev. D 91, 064049 (2015). https://doi.org/10.1103/PhysRevD.91. 064049. arXiv:1411.2386 [gr-qc]

86. S .H. Hendi, B. Eslam Panah, S. Panahiyan, Einstein-BornInfeld-massive gravity: AdS-black hole solutions and their thermodynamical properties. JHEP 1511, 157 (2015). https://doi.org/ 10.1007/JHEP11(2015)157. arXiv:1508.01311 [hep-th]

87. M. Kord Zangeneh, A. Dehyadegari, M .R. Mehdizadeh, B. Wang, A. Sheykhi, Thermodynamics, phase transitions and Ruppeiner geometry for Einstein-dilaton-Lifshitz black holes in the presence of Maxwell and Born-Infeld electrodynamics. Eur. Phys. J. C 77(6), 423 (2017). https://doi.org/10.1140/epjc/ s10052-017-4989-0. arXiv:1610.06352 [hep-th]

88. X.X. Zeng, X.M. Liu, L.F. Li, Phase structure of the BornInfeld-anti-de Sitter black holes probed by non-local observables. Eur. Phys. J. C 76(11), 616 (2016). https://doi.org/10.1140/epjc/ s10052-016-4463-4. arXiv:1601.01160 [hep-th]

89. S. Li, H. Lu, H. Wei, Dyonic (A)dS black holes in Einstein-Born-Infeld theory in diverse dimensions. JHEP 1607, 004 (2016). https://doi.org/10.1007/JHEP07(2016)004. arXiv:1606.02733 [hep-th]

90. S. Hossein Hendi, B. Eslam Panah, S. Panahiyan, M. Hassaine, BTZ dilatonic black holes coupled to Maxwell and Born-Infeld electrodynamics. Phys. Rev. D 98(8), 084006 (2018). https://doi.org/10.1103/PhysRevD.98.084006. arXiv: 1712.04328 [physics.gen-ph]

91. A. Dehyadegari, A. Sheykhi, Reentrant phase transition of Born-Infeld-AdS black holes. Phys. Rev. D 98(2), 024011 (2018). https://doi.org/10.1103/PhysRevD.98.024011. arXiv: 1711.01151 [gr-qc]

92. S.H. Hendi, M. Momennia, Reentrant phase transition of Born-Infeld-dilaton black holes. Eur. Phys. J. C 78(10), 800 (2018). https://doi.org/10.1140/epjc/s10052-018-6278-y. arXiv: 1709.09039 [gr-qc]

93. P. Wang, H. Wu, H. Yang, Thermodynamics and phase transition of a nonlinear electrodynamics black hole in a cavity. arXiv:1901.06216 [gr-qc]

94. H. Benbellout, J. Diaz-Alonso, D. Rubiera-Garcia, Structure and thermodynamics of charged nonrotating black holes in higher dimensions. Phys. Rev. D 99(8), 084027 (2019). https://doi.org/ 10.1103/PhysRevD.99.084027. arXiv:1807.06298 [gr-qc] 
95. B. Mu, P. Wang, H. Yang, Covariant GUP deformed HamiltonJacobi method. Adv. High Energy Phys. 2017, 3191839 (2017). https://doi.org/10.1155/2017/3191839. arXiv:1408.5055 [gr-qc]

96. J. Tao, P. Wang, H. Yang, Black hole radiation with modified dispersion relation in tunneling paradigm: static frame. Nucl. Phys. B 922, 346 (2017). https://doi.org/10.1016/j.nuclphysb.2017.06. 022. arXiv:1505.03045 [gr-qc]

97. R. Pellicer, R.J. Torrence, Nonlinear electrodynamics and general relativity. J. Math. Phys. 10, 1718 (1969). https://doi.org/10.1063/ 1.1665019

98. J. Plebanski, Non-linear electrodynamics. a study (Monograph of CINVESTAV, Mexico City, 1966)
99. E. Jamsin, A note on conserved charges of asymptotically flat and anti-de Sitter spaces in arbitrary dimensions. Gen. Relativ. Gravit. 40, 2569 (2008). https://doi.org/10.1007/s10714-008-0640-6. arXiv:0705.0484 [gr-qc]

100. D. Kastor, S. Ray, J. Traschen, Smarr formula and an extended first law for lovelock gravity. Class. Quantum Gravity 27, 235014 (2010). https://doi.org/10.1088/0264-9381/27/23/ 235014. arXiv:1005.5053 [hep-th]

101. Y.W. Han, M.J. Lan, X.X. Zeng, Thermodynamics and weak cosmic censorship conjecture in (2+1)-dimensional regular black hole with nonlinear electrodynamics sources. arXiv:1903.03764 [gr-qc] 